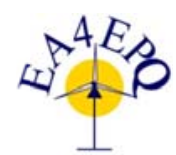

International Conference on Renewable Energies and Power Quality (ICREPQ'16)

Madrid (Spain), $4^{\text {th }}$ to $6^{\text {th }}$ May, 2016

Renewable Energy and Paver Quality. Tournal (RE\&PQJ)

ISSN 2172-038 X, No.14 May 2016

\title{
Voltage/load Control Strategy for Smart Distribution Grids with a High Penetration of Renewable Energy Sources
}

\author{
NourEldeen Elsayad ${ }^{1}$, Khaled Bashir Shaban ${ }^{1}$, Ahmad Massoud ${ }^{1}$ and Ramadan ElShatshat ${ }^{2}$ \\ ${ }^{1}$ Computer Science and Engineering Department, \\ College of Engineering, \\ Qatar University \\ ${ }^{2}$ Department of Electrical and Computer Engineering \\ University of Waterloo \\ raelshatshat@uwaterloo.ca
}

\begin{abstract}
The exponential increase of renewable energy sources (RESs) penetration in the power grid introduces the of voltage instability when RESs are integrated into medium and low voltage networks. This paper proposes a state-machine based voltage/load control strategy for different operating scenarios in a system comprised of a large RES power plant connected to a medium voltage bus at $11 \mathrm{kV}$, and small RES systems scattered across low voltage networks at $415 \mathrm{~V}$. Three modes of operation are summarized, in which the appropriate action is taken according to the availability of RES power generation in the network and the loading condition of this network. Efficient power sharing is achieved via the utilization of a communication network deployed between the RES systems. The communication protocol of the network is represented in four messages adopted by the RES systems integrated into the grid. The effectiveness of the proposed voltage/load control strategy to surpass overvoltage cases caused by over-generation of RES power is illustrated through simulations. The proposed strategy can be adopted by either agreement between a utility and RES owners, or by making it the basis for a standard for RES installation.
\end{abstract}

\section{Key words}

Smart grid, distribution power networks, renewable energy sources, communication networks, load power sharing, voltage regulation.

\section{Introduction}

Distributed energy sources are receiving wide acceptance, especially with the emergence of systems that harvest renewable resources, e.g., solar and wind, as they are sustainable, pollution-free, stationary and incorporate modularity features. Having renewable energy sources (RESs), e.g., photovoltaics (PVs) and windmills, dispersed throughout the power network coincides with the growing interest in equipping power grids with selfhealing technologies to autonomously reroute power flow during faults and minimize the number of loads affected by power outages [1]-[4].

In the last decade, the world's interest in RES systems has exponentially increased. As an example, the capacity of integrated PV systems has increased from $9.2 \mathrm{GW}$ in 2007 to more than $102 \mathrm{GW}$ in 2012 [5, 6], and the International
Energy Agency predicts that the solar energy sources will exceed $11 \%$ of world electricity generation by 2050 [5]. Many studies point the fact that the steadily increasing penetration of distributed RES systems in power networks worldwide will lead to technical challenges and could cause problems [7, 8]. For instance, overvoltage, if it is not well-managed, in affecting power delivery reliability and diminishing the benefits gained from investments in RES systems [9-13].

Many approaches have been proposed to address the problem of overvoltage due to RES systems' high penetration of power networks. One approach relies on upgrading the elements and devices of the power networks to be more tolerant to overvoltage limits by using larger conductors and transformers [13, 17]. However, this approach increases the cost of RES system adoption by the power grid. Another solution utilizes the load tap changer positions of distribution transformers to confine the voltage level within allowable limits $[14,15]$, but this approach does not cope very well with the continuously changing power generation nature of RES systems. Thus, the tap changers will degrade and require maintenance [16]. A third way to resolve over-voltage issue involves reactive power compensation $[15,17]$. Installing a reactor to consume reactive power from a system does indeed reduce the voltage magnitude to an acceptable level, but this solution is costly [18].

The active component of the distribution networks' impedance is relatively higher than the reactive component, i.e., $\mathrm{R} / \mathrm{X}$ is high, giving the real power flowing in the network more influence in the voltage profile [19]. Consequently, manipulating the real power injected into the grid is a very effective way to control the voltage magnitude at the points of common coupling between an RES system and a grid [20, 21]. Real power regulation does not need any upgrade in the network or the interfacing systems of RES systems for reactive power compensation; all these features make this an approach favored by utilities and customer [17]. 
Many real power management techniques have been investigated and proposed to solve the issue of voltage rise due to high RES penetration. One of these techniques utilizes energy storage systems to consume any excess in generated renewable power and thus control voltage magnitude [22]. Another way to manage real power injected into the grid is active power drop control; this technique is used in distribution networks when the $\mathrm{R} / \mathrm{X}$ ration is high $[17,19]$. Many studies have also investigated the utilization of sensitivity methods to manage voltage profiles [23, 24].

This paper proposes a smart state-machine based voltage/load control strategy suitable for RES embedded in distribution systems. The proposed strategy encompasses two operation schemes for addressing voltage rise problems. The first scheme is based on a local solution wherein a low voltage (LV) RES solves, on its own, overvoltage incidents at the nearby bus by reducing its generation. The second is a global and collaborative solution whereby other RESs connected to the LV network decrease their generation in order to reduce the voltage level at the bus suffering overvoltage. The proposed solution utilizes a communication network deployed to exchange information between the scattered RESs and so optimize the contribution of each in supplying the loads with power. In this work, it is assumed that all controlled RESs are owned by the same utility.

The rest of the paper is organized as follows. Different system-operation scenarios under different load conditions are discussed in Section II. The proposed control strategy is demonstrated in Section III. Simulation results are presented in Section IV. Section V concludes the paper.

\section{Operation Scenarios of RES}

This section studies system behavior under different loading conditions and RES power generation capabilities. The system depicted in Figure 1 is considered as the case under study. This system is comprised of a large RES power plant connected to a power grid at the $11 \mathrm{kV}$ bus, and dispersed RES at the $415 \mathrm{~V}$ buses. $\mathrm{P}_{\mathrm{G}}$ is the power supplied by the grid to feed the loads downstream the $11 \mathrm{kV}$ bus. $\mathrm{P}_{\mathrm{RES}}$ is the power fed from the large RES power plant connected to the $11 \mathrm{kV}$ bus, while $\mathrm{P}_{\text {RES1 }}, \mathrm{P}_{\mathrm{RES} 2}, \ldots$, $\mathrm{P}_{\mathrm{RESn}}$ are the injected power of the RES systems connected to the $415 \mathrm{~V}$ buses. $\mathrm{P}_{\mathrm{L} 1}, \mathrm{P}_{\mathrm{L} 2}, \ldots, \mathrm{P}_{\mathrm{Ln}}$ are the power consumed by the loads connected to the network at the low voltage side of the secondary transformer. It is assumed that the RESs are working in the maximum power point tracking (MPPT) mode, where they inject the maximum power they can generate into the grid. However, according to the loads connected to the network, the power that can be generated by the RESs, and the load buses' voltages, the operation of these RESs may be changed from the MPPT mode to other modes of operation to avoid over-voltage situations. For simplicity, it is also important to note that it has been assumed that the network, under study contains neither power storage nor voltage/VAR control devices. For this system to operate, it relies on the power generation capability of these RES systems, and the power demand of the network.
Accordingly, four scenarios of operation are discussed next.

A. First Scenario

Normally, the total RES power generation from both the distributed LV RES and the MV large RES power plant is less than the load demand downstream the $11 \mathrm{kV}$ bus, and the generation of each LV RES is less than the local demand of the load connected to the same LV bus. In this case, the LV load buses will operate at their maximum capability without causing any problems to the voltage profile.

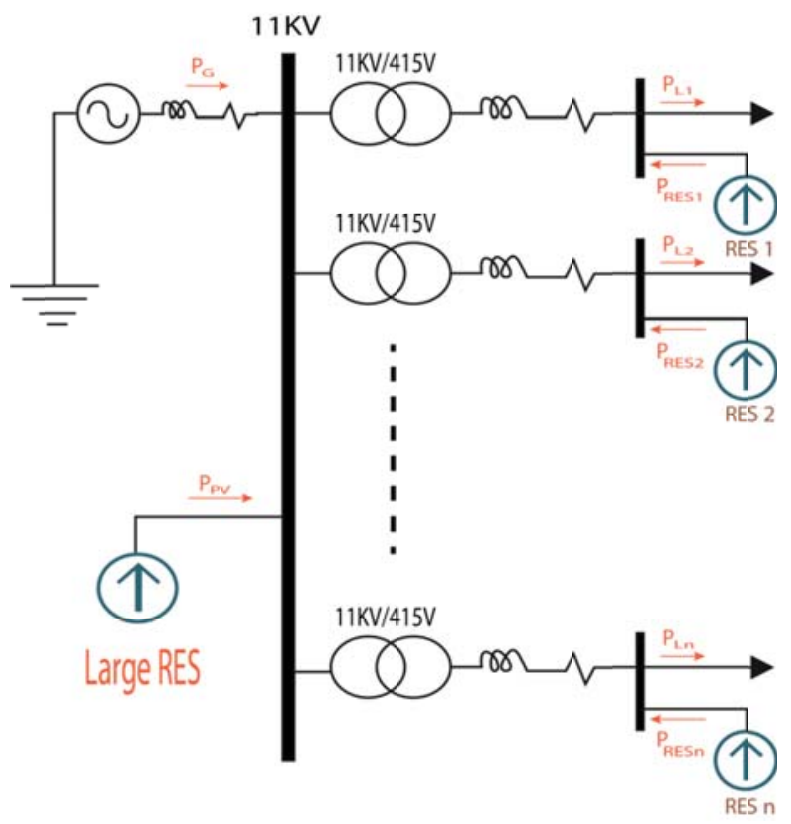

Figure 1 Medium and low voltage power networks under study

\section{B. Second Scenario}

In this scenario, the total power generated from both RES systems is less than the load demand downstream the $11 \mathrm{KV}$ bus, but the generation of some of the distributed LV RES systems surpasses the local demand of the load connected to their LV buses. In this case, the excess in the generated RES power will be injected upstream their LV buses, causing the voltage magnitude to rise.

\section{Third Scenario}

This scenario occurs when the total RES power generation from both the distributed and the large RES systems surpasses the load demand downstream the $11 \mathrm{KV}$ bus, but the generation of each LV RES system is less than the local demand of the load connected to the same LV bus. In this case, the RES power generated from the MV large RES power plant gets injected into the grid, downstream the $11 \mathrm{KV}$ bus.

\section{Fourth Scenario}

In this case, the total RES power generation from both the distributed LV RES and the MV large RES power plant surpasses the load demand downstream the $11 \mathrm{KV}$ bus, and the generation of each of the distributed LV RES systems exceeds the local demand connected to the same LV bus. When this scenario develops, the excess in the generated RES power from both the LV distributed RES and the MV large RES power plant gets injected into the grid, upstream the $11 \mathrm{KV}$ bus, which in its turn raises the voltage magnitude on all the LV load buses. 


\section{The Proposed Control Strategy}

This section is divided into three main parts: the first describes the operation modes of both the LV RES and MV RES systems, the second illustrates the proposed communication networking between the RES systems, and the third describes the control scheme in the form of state machines.

\section{A. Operation Modes}

As discussed in the previous section, the operation of the RES systems connected to the network depends on the loading condition of the network and the power that can be generated by the RES. Accordingly, the operation of each individual RES can be classified into three modes of operation:

- $\quad$ Mode 1: this is the MPPT mode of operation, where the RES injects the maximum active power it can generate into the grid. This is the default mode of operation for the RES when demand is relatively larger than the generated RES power.

- Mode 2: in this mode, the RES controller turns off the maximum power point tracker, and tries to inject the adequate active power required to hold the voltage at the point of common coupling (PCC), $\mathrm{V}_{\mathrm{PCC}}$, at a reference level. The RES enters this mode when the voltage at the PCC exceeds the allowable voltage limit.

- Mode 3: here, the LV RESs reduce the active power injected into the network by a certain level, $\Delta \mathrm{P}$ (this power reduction amount differs according to the capacity of the renewable energy source) after it receives a command from the large RES power plant. This mode is designated to increase the contribution of the large RES power plant at the $11 \mathrm{kV}$ bus in supplying the load power, through maximum power point operation if possible, in order to relieve tension on the lightly loaded buses in the network.

\section{B. Communication Protocol}

As discussed under the fourth RES operation scenario, in some cases, the local solution of changing the mode of operation from mode 1 to mode 2 , in order to limit the RESs injected power to the PCC at a certain bus in the network, may not be enough to reduce $\mathrm{V}_{\mathrm{PCC}}$. Therefore, communication should occur between the distributed RES at the $415 \mathrm{~V}$ buses and the large RES power plant at the $11 \mathrm{kV}$ bus, in order to control the flow of RES power fed to the network, and to relieve the tension on the very lightly loaded buses without affecting the operation of the large RES power plant. For this, we propose two-way communication between the large RES power plant at the $11 \mathrm{kV}$ bus and the distributed LV RES systems. This communication system should not be expensive or complex, i.e., it should be scalable as the number of RESs increase. Moreover, it should have a standard communication protocol to be adopted by RESs integrated into the network. This system allows a distributed RES to transmit/receive commands and alerts to/from the large RES power plant.
The data exchanged between the distributed RES systems and the large RES power plant can be summarized into four messages, as follows:

- M1: This message is sent to the large RES power plant by any distributed RES that experiences overvoltage and the local solution, changing the mode of operation from mode 1 to mode 2 , has failed to reduce the bus voltage to an acceptable level. This message is considered as a request to either find another path for the power coming from the large RES plant, or to reduce the power this RES power plant injects into the network so as to regulate the voltage on that lightly loaded bus to the acceptable range.

- M2: This message is sent by the large RES power plant to the distributed RESs, in order to reduce their respective injected active power to the grid. Each RES that receives this message has to reduce its injected active power by $\Delta \mathrm{P}$. Adequate injected power reduction by the distributed RES may require the large RES power plant to send the M2 message many times until the system reaches a point where the RES power flow does not cause over-voltage on any of the network buses. Otherwise, the large RES plant will have to reduce its generation of power.

- M3: This is the distributed RES response to an M2 message, when the RES has reduced its injected power by $\Delta \mathrm{P}$.

- M4: This is the distributed RES response to an M2 message when the RES cannot reduce its injected power by $\Delta \mathrm{P}$, because the RES is already in a state of operation and has already injected active power of $<$ $\Delta \mathrm{P}$ to the grid..

\section{State-machine based Control Strategy}

Figures 2 and 3 show the state-machines of the proposed operation control strategy of the distributed LV RES system and the large MV RES power plant, respectively. The distributed LV RES has the capability to move between the three modes of operation, depicted by circles. The transitions between the different modes of operation, depicted by arrows, depend on the network load conditions, the RES power generation, and the messages received from the large RES power plant. In the proposed control strategy, there are six transitions, as shown in Figure 2. The transitions between the three modes occur as follows:

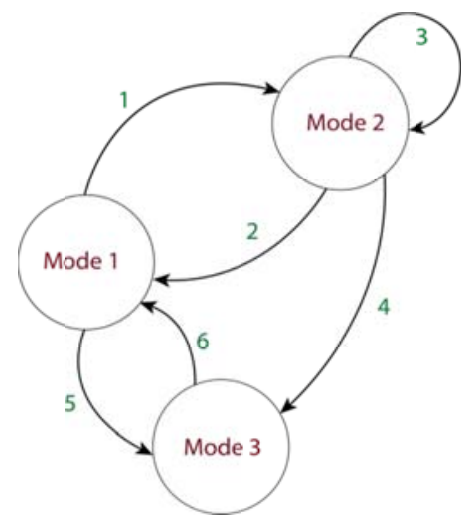

Figure 2. Distributed LV RES state machine. 


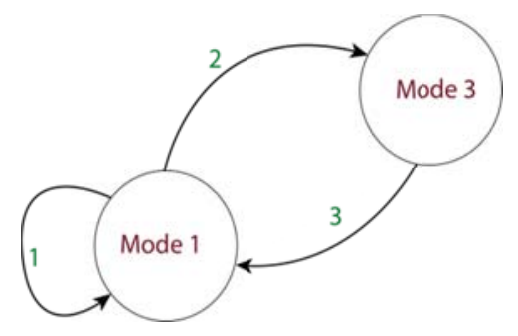

Figure 3. MV RES power plant state machine.

- Transition (1): when the LV RES is in mode 1 and an overvoltage is detected, the system changes to operation mode 2 , in an attempt to reduce the bus voltage to an acceptable level.

- Transition (2): when the LV RES is in mode 2 and experiences an under-voltage, the system returns to mode 1 , where it injects the maximum active power it can harvest, and injects the required reactive power to compensate for the voltage drop.

- Transition (3): when the LV RES is in mode 2, if it attempts to keep the load bus voltage at an acceptable level by reducing its RES power generation, but fails to fulfill this condition, the system stays in mode 2 , where its injected active power is $\approx 0$, and sends the M1 message.

- Transition (4): when the LV RES is in mode 2 and receives the M2 message, it changes to operation mode 3, where it reduces its injected RES power by $\Delta \mathrm{P}$.

- Transition (5): when the LV RES is in mode 1 and receives the M2 message, it changes to mode of operation 3, where it turns off the maximum power point tracker, and reduces its injected RES power by $\Delta \mathrm{P}$.

- Transition (6): after the system moves to mode 3, a timer starts counting, and when the counted time equals $T_{R}$, the system returns to mode 1 . This technique relies on making a perturbation in the system and observing the consecutive outcome. If the load has changed in that time $T_{R}$ and the network can tolerate the RES power generated in mode 1 , no problem is faced. Otherwise, the system moves to mode 2 , and settles, or returns to mode 3.

Figure 3, depicts the state machine of the control strategy proposed for the large MV RES power plant in our system. The state machine comprises two modes of operation, mode1, and mode 3 , and three transitions. These transitions between the two modes occur as follows:

- Transition (1): when the large RES power plant is under normal operation, in mode 1 , and receives the M1 message, it stays in mode 1, and sends the M2 message to all the distributed RESs. When it receives M3 messages among the responses from the distributed RESs, this means that at least some of them have taken action and reduced their injected power. If the MV RES power plant receives the M1 message again, the problem still exists, and at this time, the MV RES power plant will stay in mode 1 and send the M2 message again. This process will repeat as long as the MV RES power plant keeps receiving M1 messages and receiving the M3 response from the distributed LV RES.

- Transition (2): As a completion to the scenario discussed in the previous transition, when the large MV RES power plant receives the message M1, but all the responses from the distributed LV RES are the M4 message, the network is very lightly loaded, and even though the distributed LV RES are not injecting any power to the network, some of the loads are experiencing an overvoltage because the network cannot tolerate the RES power generated by the large MV RES plant when it is operating in mode 1. At this moment, the MV RES power plant will be coerced to operate in mode 3 , in order to reduce its power generation to a level that can be tolerated by the network.

- Transition (3): As discussed in the sixth transition in the distributed LV RES, where a perturbation occurs after a time $T_{K}$, to restore the MV RES plant to mode 1 , then observe whether the network after that time has become better able to tolerate the power coming from the MV RES plant when it is operating in mode 1 or not.

\section{Simulation Results}

The system under study and the proposed control strategy are simulated using MATLAB/SIMULINK. Figure 4 depicts the system, wherein the grid infinite bus is connected to the $11 \mathrm{kV}$ bus, via $\mathrm{R}_{1}$ and $\mathrm{L}_{1}$, representing the equivalent impedance of the MV network. The large RES system is connected directly to the $11 \mathrm{kV}$ bus. Two radial feeders are connected to the LV load buses. The first radial system, with load1, is the system where three operation scenarios are investigated in this section. The other radial system depicts collectively the network's other radial systems connected to the $11 \mathrm{KV}$ bus. The parameters of the system under study are in Table I.

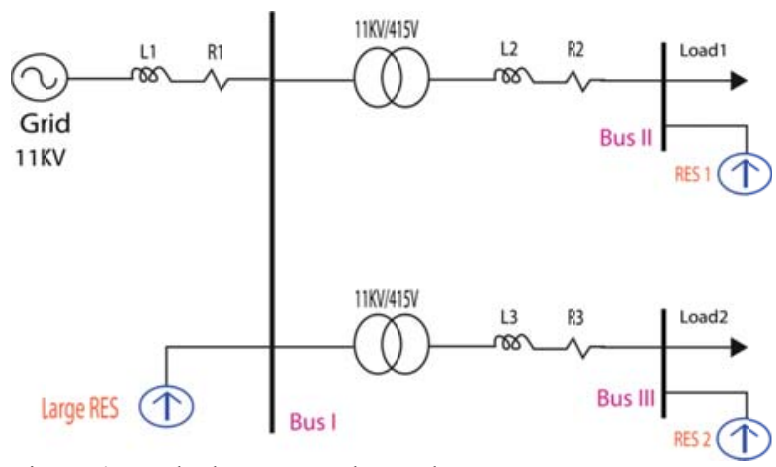

Figure 4. Testbed system under study.

Table I. Testbed System Parameters

\begin{tabular}{|l|l|}
\hline Parameters & Value \\
\hline R1 $(\Omega / \mathrm{km})$ & 0.17 \\
\hline L1 $(\mathrm{mH} / \mathrm{km})$ & 1.6 \\
\hline Length of feeder $1(\mathrm{~km})$ & 3 \\
\hline R2 $(\Omega / \mathrm{km})$ & .17 \\
\hline L2 $(\mathrm{mH} / \mathrm{km})$ & .83 \\
\hline Length of feeder $2(\mathrm{~km})$ & 6 \\
\hline R3 $(\Omega / \mathrm{km})$ & 0.2 \\
\hline L3 $(\mathrm{mH} / \mathrm{km})$ & 1 \\
\hline Length of feeder 3 $(\mathrm{km})$ & 2 \\
\hline
\end{tabular}


Figure 5 shows the impact of light loading on the voltage profile at bus II, V2, when load 1, Pload1, is reduced at $\mathrm{t}=$ $2 \mathrm{sec}$. At that time, bus II experiences a rise in its voltage, which in our case has exceeded the allowable limit. When the load is reduced, the excess in the generated RES power at bus II, PRES1, is injected back to the MV network, causing an increase in the magnitude of V2, which may result in a severe damage to these loads. At $\mathrm{t}=$ $2.5 \mathrm{sec}$, the local solution is applied, by reducing the RES power generation at bus II, PRES1, which results in a decrease in V2 magnitude. In this case, the local solution is sufficient to return the bus voltage to the acceptable limit, and no global solution is needed.
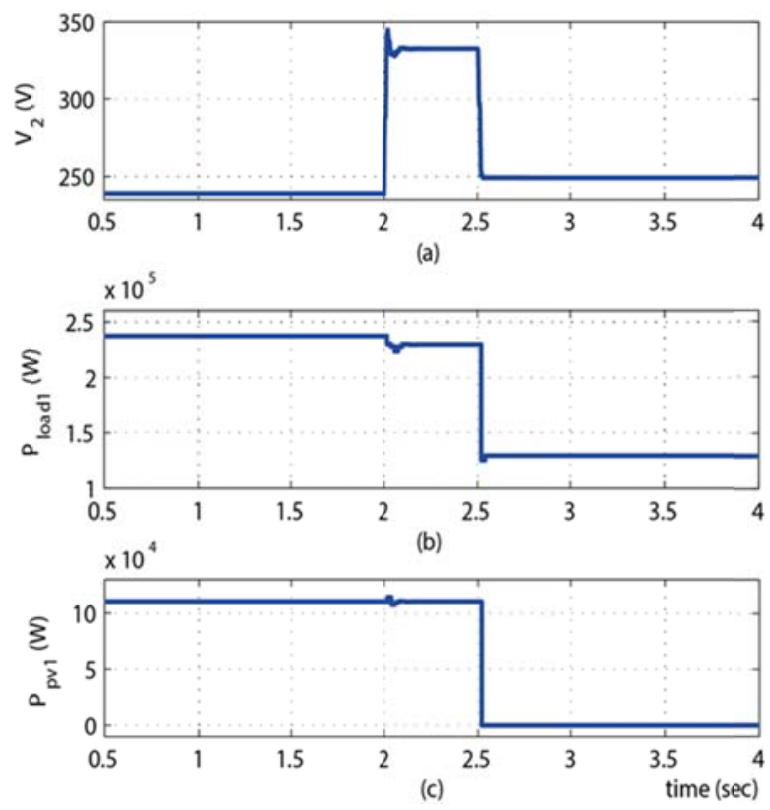

Figure 5. Impact of load change on the voltage profile at the LV bus and the result of applying the local solution.

Figure 6, shows a different scenario wherein the local solution is not enough to alleviate the overvoltage problem at bus II, caused by light loading. In this case, a global solution takes place, which requests the other LV RESs, connected to other radial feeders, to reduce their generation. The impact of reducing the RES power generation at bus III, PRES2, on V2, is shown in Figure 6. The reduction in V2 is a result of returning RES power to the grid through the MV network, PG. In this global solution, the controller does not reduce the RES power generated from the large RES power plant at the $11 \mathrm{KV}$ bus, PRES, and only manipulates the RES generation of the LV RES. When this first global solution, shown in Figure 6, fails to return V2 to the allowable voltage range, another global, albeit collaborative, solution takes place.

This second solution relies on reducing the RES generation from the large RES power plant at bus I, $\mathrm{P}_{\mathrm{RES}}$. Figure 7 shows the voltage profile at bus II, V2, after applying the first external solution at $\mathrm{t}=2 \mathrm{sec}$. When the first global solution fails to return V2 to an acceptable magnitude, the second global solution is applied, by reducing $\mathrm{P}_{\text {RES }}$ generation at $\mathrm{t}=2.8 \mathrm{sec}$. Switching between the local and the global solutions is based on the proposed messaging system. The controller starts solving the overvoltage problem at any LV bus by the local solution. When the local solution does not bring the load bus voltage to the acceptable range, the controller switches to the first global solution. Eventually, if the first global solution is not adequate, the controller switches to the second external solution.
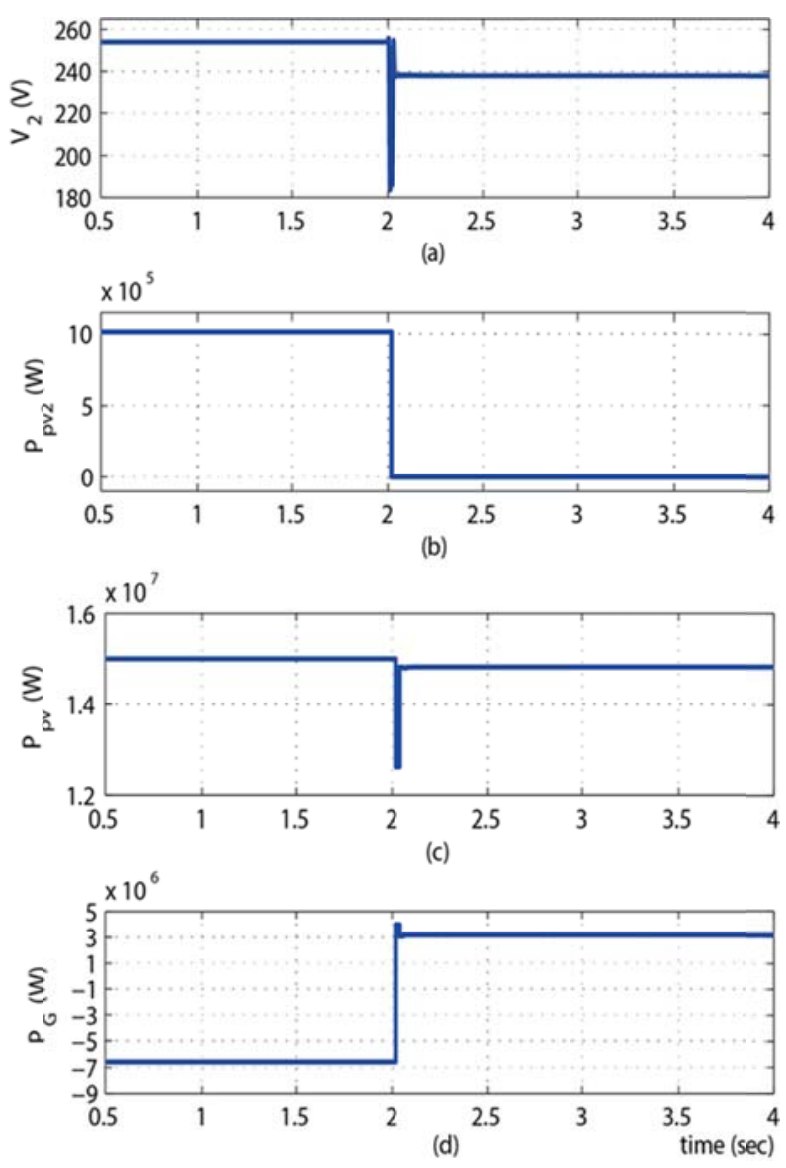

Figure 6. The impact of applying the first global solution.

\section{Conclusion}

A control strategy of RES systems operating in parallel to feed the network loads with power is proposed in this paper. The strategy assumes a communication network in place that facilitates exchanging four messages between the RES sources. The MV large RES power plant can command the distributed RESs to reduce their generation of power when the network is lightly loaded, thus giving room for the MV large RES power plant to supply the network with its maximum power capability, which is more economically beneficial than reducing its power generation.

\section{Acknowledgement}

This work was made possible by NPRP 6-711-2-295 grant from the Qatar National Research Fund (a member of Qatar Foundation). The statements made herein are solely the responsibility of the authors. 

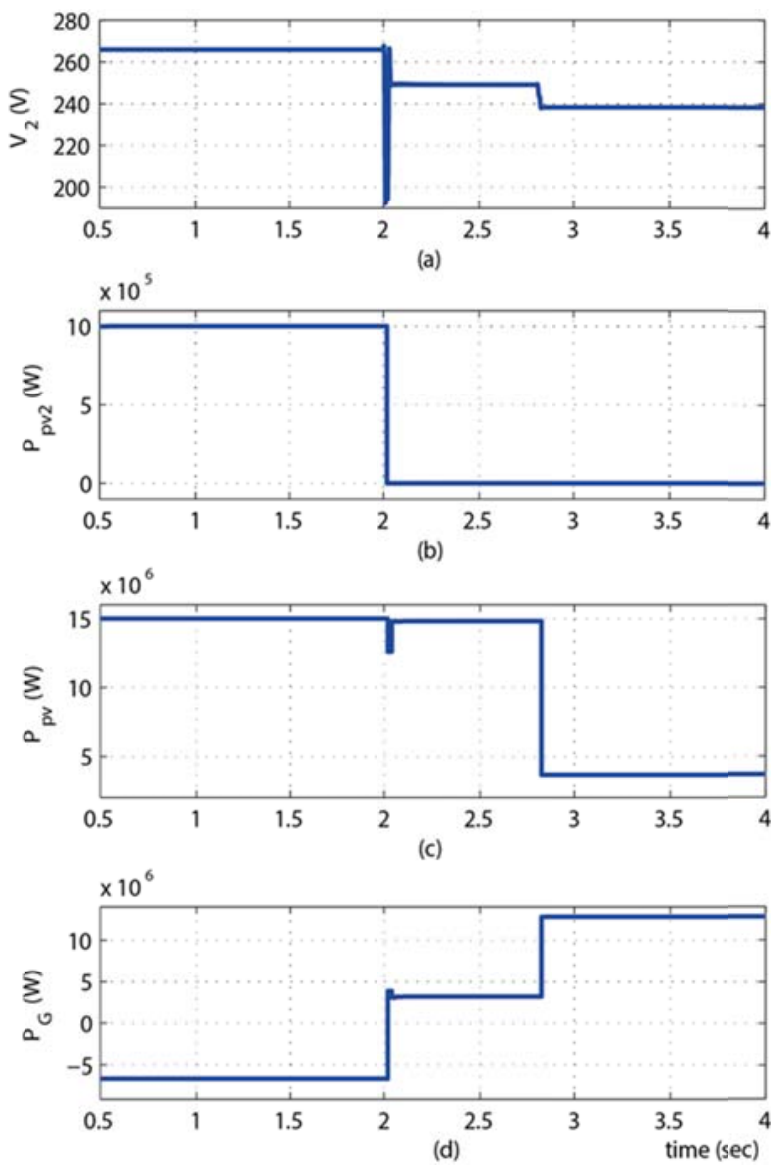

Figure 7. The impact of applying the second global solution.

\section{References}

[1] Haoming Liu; Xingying Chen; Kun Yu; Yunhe Hou, "The Control and Analysis of Self-Healing Urban Power Grid," in Smart Grid, IEEE Transactions on, vol. 3, no. 3, pp.1119-1129, Sept. 2012.

[2] Zaibin Jiao; Xiaobing Wang; Heteng Gong, "Wide area measurement/wide area information-based control strategy to fast relieve overloads in a self-healing power grid," in Generation, Transmission \& Distribution, IET, vol. 8, no. 6, pp.1168-1176, June 2014.

[3] Juan Li; Chen-Ching Liu; Schneider, K. P., "Controlled Partitioning of a Power Network Considering Real and Reactive Power Balance," in Smart Grid, IEEE Transactions on, vol. 1, no. 3, pp. 261-269, Dec. 2010.

[4] Arefifar, S. A.; Mohamed, Y. A. I.; El-Fouly, T. H. M., "SupplyAdequacy-Based Optimal Construction of Microgrids in Smart Distribution Systems," in Smart Grid, IEEE Transactions on, vol. 3, no. 3, pp. 1491-1502, Sept. 2012.

[5] Moosavian, S. M.; Rahim, N. A.; Selvaraj, J.; Solangi, K. H., "Energy policy to promote photovoltaic generation," in Renewable and Sustainable Energy Review, vol. 25, pp. 44-58, Sep. 2013.

[6] Masson, G.; Latour, M.; Rekinger, M., Theologitis, I. T.; Papoutsi, M., "Global Market Outlook for Photovoltaics 2013-2017," European Photovoltaic Industry Association, [Online].

[7] von Appen, J.; Braun, M.; Stetz, T.; Diwold, K.; Geibel, D., "Time in the Sun: The Challenge of High PV Penetration in the German Electric Grid," in Power and Energy Magazine, IEEE, vol. 11, no. 2, pp.55-64, March-April 2013.

[8] Summary Report for Utility Experience With High Penetration PV Workshop [Online], December 2012. Available: http://energy.sandia.gov/wp/wpcontent/gallery/uploads/High-PenPVWorkshop-at-IRED.pdf

[9] Thomson, M.; Infield, D. G., "Network Power-Flow Analysis for a High Penetration of Distributed Generation," in Power Systems, IEEE Transactions on, vol. 22, no. 3, pp. 1157-1162, Aug. 2007.

[10] Coster, E. J.; Myrzik, J. M. A.; Kruimer, B.; Kling, W. L., "Integration Issues of Distributed Generation in Distribution
Grids," in Proceedings of the IEEE, vol. 99, no. 1, pp. 28-39, Jan. 2011.

[11] Chao-Shun Chen; Chia-Hung Lin; Wei-Lin Hsieh; Cheng-Ting $\mathrm{Hsu}$; Te-Tien Ku; Chin-Ying Ho, "Effect of load transfer to penetration level of PV generation in distribution system," in Innovative Smart Grid Technologies - Asia (ISGT Asia), 2012 IEEE, pp. 1-6, 21-24 May 2012.

[12] Chia-Hung Lin; Wei-Lin Hsieh; Chao-Shun Chen; Cheng-Ting Hsu; Te-Tien Ku; Cheng-Ta Tsai, "Financial Analysis of a LargeScale Photovoltaic System and Its Impact on Distribution Feeders," in Industry Applications, IEEE Transactions on, vol. 47, no. 4, pp. 1884-1891, July-Aug. 2011.

[13] Yang Wang; Peng Zhang; Wenyuan Li; Weidong Xiao; Abdollahi, A., "Online Overvoltage Prevention Control of Photovoltaic Generators in Microgrids," in Smart Grid, IEEE Transactions on, vol. 3, no. 4, pp. 2071-2078, Dec. 2012.

[14] Hazel, T. G.; Hiscock, N.; Hiscock, J., "Voltage Regulation at Sites With Distributed Generation," in Industry Applications, IEEE Transactions on, vol. 44, no. 2, pp. 445-454, March-April 2008.

[15] Tengku Hashim, T. J.; Mohamed, A.; Shareef, H., "A review on voltage control methods for active distribution networks," PRZEGLAD ELEKTROTECHNICZNY (Electrical Review), vol. 88, pp. 304-312, Jun. 2012.

[16] IEC, Tap-Changers-Part 2: Application Guide, IEC Standard 60214-2,2004

[17] Tonkoski, R.; Lopes, L. A. C.; El-Fouly, T. H. M., "Coordinated Active Power Curtailment of Grid Connected PV Inverters for Overvoltage Prevention," in Sustainable Energy, IEEE Transactions on, vol. 2, no. 2, pp. 139-147, April 2011.

[18] Alyami, S.; Yang Wang; Caisheng Wang; Junhui Zhao; Bo Zhao, "Adaptive Real Power Capping Method for Fair Overvoltage Regulation of Distribution Networks With High Penetration of PV Systems," in Smart Grid, IEEE Transactions on, vol. 5, no. 6, pp. 2729-2738, Nov. 2014.

[19] Tonkoski, R.; Lopes, L. A. C., "Voltage Regulation in Radial Distribution Feeders with High Penetration of Photovoltaic," in Energy 2030 Conference, 2008. ENERGY 2008. IEEE, pp. 1-7, 17-18 Nov. 2008.

[20] Smith, J.W.; Sunderman, W.; Dugan, R.; Seal, B., "Smart inverter volt/var control functions for high penetration of $\mathrm{PV}$ on distribution systems," in Power Systems Conference and Exposition (PSCE), 2011 IEEE/PES, pp. 1-6, 20-23 March 2011.

[21] Demirok, E.; Sera, D.; Teodorescu, R.; Rodriguez, P.; Borup, U., "Evaluation of the voltage support strategies for the low voltage grid connected PV generators," in Energy Conversion Congress and Exposition (ECCE), 2010 IEEE, pp.710-717, 12-16 Sept. 2010.

[22] Alam, M. J. E.; Muttaqi, K. M.; Sutanto, D., "Mitigation of Rooftop Solar PV Impacts and Evening Peak Support by Managing Available Capacity of Distributed Energy Storage Systems," in Power Systems, IEEE Transactions on, vol. 28, no. 4, pp. 38743884, Nov. 2013.

[23] Ayres, H. M.; Freitas, W.; de Almeida, M. C.; da Silva, L. C. P., "Method for determining the maximum allowable penetration level of distributed generation without steady-state voltage violations," in Generation, Transmission \& Distribution, IET, vol. 4, no. 4, pp. 495-508, April 2010.

[24] Li Yu; Czarkowski, D.; de Leon, F., "Optimal Distributed Voltage Regulation for Secondary Networks With DGs," in Smart Grid, IEEE Transactions on, vol. 3, no. 2, pp. 959-967, June 2012. 